\title{
Estudio comparativo de la densidad de siembra y su efecto sobre la producción de forraje y calidad nutricional de pastos perennes asociados, en condiciones alto andinas del Perú \\ Comparative study of sowing density and its effect on forage production and nutritional quality of associated perennial pastures, in high Andean conditions of Perú
}

\author{
Alberto Gilmer Arias-Arredondo ${ }^{1,4}$, César Enrique Pantoja-Aliaga ${ }^{1}$, Juancarlos Alejandro Cruz Luis ${ }^{2}$, \\ Alfonso Atanacio Carvajal ${ }^{3}$, Christian Miguel Candela Barra ${ }^{3}$, Melina López Rodriguez ${ }^{1}$ \\ ${ }^{1}$ Facultad de Ciencias Agropecuarias, Universidad Nacional Daniel Alcides Carrión. Pasco - Perú. E-mail: \\ albertogilmer@gmail.com; alberto.arias@epgunh.edu.pe. ORCID: https://orcid.org/0000-0002-6055-8722 \\ ${ }^{2}$ Dirección de Supervisión y Monitoreo en las Estaciones Experimentales Agrarias, Instituto Nacional de \\ Innovación Agraria. Lima - Perú. ORCID: https://orcid.org/0000-0003-1169-440X \\ ${ }^{3}$ Dirección General de Ganadería, Ministerio de Desarrollo Agrario y Riego. Lima - Perú. https://orcid.org/0000- \\ 0003-4732-9818 \\ ${ }^{4}$ Facultad de Ingeniería, Universidad Nacional de Huancavelica. Huancavelica - Perú.
}

\section{Resumen}

El estudio se llevó a cabo en la sierra central del Perú a 3900 m.s.n.m, en el centro experimental de Casaracra de la Universidad Nacional Daniel Alcides Carrión, donde el objetivo fue la de determinar y analizar el rendimiento forrajero y la calidad nutricional de los pastos asociadas en distintas densidades de siembra. En la producción forrajera se determinó los valores de materia verde y materia seca y el porcentaje de materia seca; en la calidad nutricional se analizó las variables nutricionales \%PT, \%FDN, $\%$ FDA, \%Ca y \%P. se utilizó un diseño completamente al azar. Los resultados para rendimiento forrajero para el tratamiento 1 en MV fue $3.54 \mathrm{~kg} / \mathrm{m}^{2}$ y MS $0.68 \mathrm{~kg} / \mathrm{m}^{2}$, en el tratamiento 2 en MV fue $2.61 \mathrm{~kg} / \mathrm{m}^{2}$ y MS $0.72 \mathrm{~kg} / \mathrm{m}^{2}$, para el tratamiento 3 en MV fue $3.07 \mathrm{~kg} / \mathrm{m}^{2}$ y MS $0.98 \mathrm{~kg} / \mathrm{m}^{2}$ y finalmente en el tratamiento 4 la MV fue $2.48 \mathrm{~kg} / \mathrm{m}^{2}$ y MS $0.93 \mathrm{~kg} / \mathrm{m}^{2}$. La calidad nutricional para los tratamientos1, 2,3 y 4 en \%PT $(15.56,15.21,6.81$ y 5.37), \%FDN $(48.85,43.82,43.34$ y 41.89$)$, \%FDA $(28.82,26.31$, 27.27 y 24.43$), \% \mathrm{Ca}(0.86,0.80,0.33$ y 0.17$), \% \mathrm{P}(0.25,0.24,0.18$ y 0.07$)$ respectivamente. Donde se concluye que el tratamiento 4 mostro contenidos aceptables de fibras en la alimentación de ovinos.

Palabras claves: Pasto permanente, rendimiento forrajero, calidad nutricional.

\begin{abstract}
The study was carried out in the central highlands of Peru at 3,900 meters above sea level, in the Casaracra experimental center of the Daniel Alcides Carrión National University, where the objective was to determine and analyze the forage yield and nutritional quality of the associated grasses in different density is sowing. In forage production, the values of green matter and dry matter and the percentage of dry matter were determined; in nutritional quality, the nutritional variables\% $\mathrm{PT}, \% \mathrm{NDF}$, $\% \mathrm{FDA}, \% \mathrm{Ca}$ and $\% \mathrm{P}$ were analyzed. a completely randomized design was used. The results for forage yield for treatment 1 in MV was $3.54 \mathrm{~kg} / \mathrm{m}^{2}$ and DM $0.68 \mathrm{~kg} / \mathrm{m}^{2}$, in treatment 2 in MV it was $2.61 \mathrm{~kg}$ $/ \mathrm{m}^{2}$ and DM $0.72 \mathrm{~kg} / \mathrm{m}^{2}$, for treatment 3 in MV it was $3.07 \mathrm{~kg} / \mathrm{m}^{2}$ and DM $0.98 \mathrm{~kg} / \mathrm{m}^{2}$ and finally in treatment 4 the MV was $2.48 \mathrm{~kg} / \mathrm{m}^{2}$ and DM $0.93 \mathrm{~kg} / \mathrm{m}^{2}$. The nutritional quality for treatments 1 , 2,3 and 4 in\% PT (15.56, 15.21, 6.81 and 5.37), \% FDN (48.85, 43.82, 43.34 and 41.89), \% FDA (28.82, $26.31,27.27$ and 24.43$), \% \mathrm{Ca}(0.86,0.80,0.33$ and 0.17$), \% \mathrm{P}(0.25,0.24,0.18$ and 0.07$)$ respectively. Where it is concluded that treatment 4 showed acceptable fiber content in sheep feeding.
\end{abstract}

Keywords: Permanent pasture, forage yield, nutritional quality. 


\section{Introducción}

En la zona altoandina del Perú, la producción de forraje tiene como base los pastos naturales el cual cuenta con una extensión de 18018794 has (INEI, 2012) los cuales se encuentran en condiciones pobres y muy pobres con una baja capacidad de carga animal de 0.5 U.O/ha/año (Flores et al., 2005A; Contreras et al., 2013; Rodríguez, 2004) y de baja calidad nutricional, siendo en la época de estiaje la más crítica con bajos contenidos de proteína (7.7\%), valores inadecuados de fibra detergente neutra $(70.8 \%)$ y limita energía metabolizable de (5.2 MJ/kgMS), los cuales son considerados críticos, inferiores e inadecuados para el mantenimiento del ganado lo que conlleva a una disminución de los parámetros productivos y reproductivos de los ovinos (Flores et al., 2003; Flores et al., 2005A; NRC, 2001).

La crianza de ovinos viene a ser una actividad de vital importancia en la zona, tanto en lo económico y social, ya que estos producen carne, leche y lana, siendo de este modo el principal sustento de más de 500 mil familias campesinas (Aliaga, 2012; Flores et al., 2003). En la actualidad se vienen instalando una serie de alternativas de pastos anuales y perennes (Avena sativa, Lollium multiflorum lam, Lollium perenne, Dactylis glomerata, Trifolium repens y Trifolium pratense) con la finalidad de mejorar la producción y calidad nutricional del forraje, para así asegurar la producción del ganado durante el periodo de escasez (Duldhy et al., 1994).

La producción de forraje y calidad nutricional de pastos cultivados perennes muestran volúmenes de forraje en una asociación de Dactyles glomerata y Trifolium pratense de $6167.7 \mathrm{~kg} / \mathrm{MS} / \mathrm{ha}$ para todo el año y en la calidad nutricional muestra contenidos de proteína cruda de $16.2 \%$, fibra detergente neutro de $49.9 \%$, digestibilidad in vitro de la materia orgánica de $54.5 \%$, calcio con $1.01 \%$ y fósforo $0.26 \%$ (Flores et al., 2005B) lo cual significa que la asociación de pastos perennes permite aumentar la producción y la calidad nutricional del alimento que se ofrece a los ovinos en la zona altoandina del país. Sin embargo, se puede realizar estudios para establecer mecanismos para elevar la disponibilidad de forraje y el valor nutritivo. En este contexto, se realizó el presente estudio con el objetivo de determinar la producción de forraje y la calidad nutricional de pastos cultivados asociados (Lolium multiflorum lam, Lolium perenne, Dactylis glomerata, Trifolium repens y Trifolium pratense).

\section{Metodología}

Ubicación del lugar de estudio 
El estudio se realizó en el centro experimental de Casaracra de la Universidad Nacional Daniel Alcides Carrión, ubicado en el distrito de Paccha provincia de Yauli,

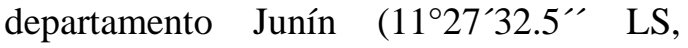
7557`25.2” LO y 3819 m.s.n.m., Arias, 2015). Donde el clima es muy frio con temperaturas que varían de entre $-1^{\circ} \mathrm{C}$ a $21^{\circ} \mathrm{C}$ para la época seca (mayo-setiembre). El suelo tiene las siguientes características un $\mathrm{pH}$ de 5.3 considerado como muy ácido, con contenidos de materia orgánica de $6.6 \%$ valores disponibles de fósforo de 2.6 ppm y potasio 142 ppm y una clase textural de franco limoso.

\section{Diseño experimental}

Se utilizó un diseño completamente al azar (DCA), con cuatro tratamientos (Tabla 1). El tamaño de cada parcela experimental fue de $2739.3 \mathrm{~m}^{2}$. Los resultados se sometieron a un ANOVA para las variables, se sometió a una prueba de medias a través de la prueba de Duncan al 5\% (Kuehl, 2000; Calzada, 1982).

Tabla 1

Formula de densidad de siembra para los tratamientos de pastos

\begin{tabular}{llcccc}
\multirow{2}{*}{\multicolumn{1}{c}{ Especie }} & \multicolumn{1}{c}{ Nombre científico } & \multicolumn{4}{c}{$\begin{array}{c}\text { Densidad de } \\
\text { siembra (kg) }\end{array}$} \\
\cline { 3 - 6 } & & $\mathbf{T - 1}^{\mathbf{a}}$ & $\mathbf{T - 2}^{\mathbf{b}}$ & $\mathbf{T - 3}^{\mathbf{c}}$ & $\mathbf{T - 4}^{\mathbf{d}}$ \\
\hline Rye grass inglés & Lolium perenne & 5.1 & 5.1 & 6.5 & 7.0 \\
Rye grass italiano & Lolium multiflorum & 3.1 & 3.5 & 4.5 & 5.5 \\
Dactyles & Dactyles glomerata & 3.1 & 3.9 & 5.0 & 7.0 \\
Trébol rojo & Trifolium pratense & 0.8 & 1.2 & 1.5 & 1.0 \\
Trébol blanco & Trifolium repens & 0.4 & 0.2 & 1.0 & 0.5 \\
\hline
\end{tabular}

${ }^{\mathrm{a}}$ Tratamiento $1,{ }^{\mathrm{b}}$ Tratamiento $2,{ }^{\mathrm{c}}$ Tratamiento $3,{ }^{\mathrm{d}}$ Tratamiento 4.

\section{Procedimiento experimental}

La preparación del terreno se realizó con un tractor agrícola iniciándose con el arado de discos a una profundidad de $25 \mathrm{~cm}$, seguido de la pasada de rastra con el fin de romper los terrones de suelos que puedan quedar para así obtener una mejor cama de siembra y maximizar el establecimiento de las semillas. Las semillas que se utilizaron fueron de la empresa Hortus S.A, el método de siembra realizado fue al voleo para cada tratamiento el cual se ejecutó el 18 de noviembre del 2015. La cosecha se realizó en el estadio de espiga de las gramíneas, el cual sucedió en el mes de mayo del 2016 después de 6 meses. Para determinar la producción de forraje se estimó los contenidos de materia verde (MV) y materia seca (MS), para MV se seleccionaron áreas de muestreo al azar utilizando un cuadrante de $1 \mathrm{~m}^{2}$ donde se realizó el corte de todas las plantas y sus partes (tallos y hojas) en total se realizaron 10 cortes considerando cada 
UNIVERSIDAD NACIONAL DE UCAYALI

Revista de Investigación Universitaria

Versión electrónica $2664-8423$

ARTICULO ORIGINAL
Vol. $11 \mathrm{~N}^{\circ} 1$, pp. 475- 482, enero/junio 2021

Recibido 16/01/2021

Aceptado 04/04/2021

Publicado 30/06/2021 una como una repetición, seguido de ello se procedió a pesar por cada corte. La MS se determinó mediante el secado de las muestras que se tenían los cuales fueron dejados al aire libre para reducir la humedad, luego de ello se llevó a la estufa a $60^{\circ} \mathrm{C}$ para completar el secado hasta obtener un peso constante de la muestra. La calidad nutricional se determinó de las muestras secas los cuales fueron triturados y molidos en un molino Wiley (malla de 1 a $2 \mathrm{~mm}$ ). Los análisis se realizaron en el laboratorio de Evaluación Nutricional de Alimentos de la Facultad de Zootecnia de la Universidad Nacional Agraria La Molina, donde se determinó los contenidos de proteína total (PT), Fibra detergente neutro (FDN), fibra detergente acido (FDA), Calcio (Ca) y Fósforo (P), los cuales fueron obtenido mediante el análisis micro Kjeldahl (Nx6.25) (AOAC, 2005). La digestibilidad in vitro se estimó por la técnica de Tilley y Terry, modificado por H.K. Van soest et al., (1991).

\section{Resultados y discusión}

\section{Rendimiento forrajero}

La variación de la producción de forraje en los diferentes tratamientos para cada variable en materia verde (MV), materia seca (MS) y la determinación del porcentaje de materia seca (\%MS) se muestran en la tabla 2. En Materia verde el tratamiento 1 mostro mayor índice de producción en comparación a los demás tratamientos 2,3 y 4 mostrando una diferencia significativa $(\mathrm{p}<0.05)$. Los tratamientos 2,3 y 4 fueron similares estadísticamente.

Tabla 2

Rendimiento forrajero de tratamientos instalados.

\begin{tabular}{lcccc}
\hline \multirow{2}{*}{\multicolumn{1}{c}{ Variable }} & \multicolumn{3}{c}{ Tratamientos } \\
\cline { 2 - 5 } & $\mathbf{T 1}^{\mathbf{a}}$ & $\mathbf{T 2}^{\mathbf{b}}$ & $\mathbf{T 3}^{\mathbf{c}}$ & $\mathbf{T 4}^{\mathbf{d}}$ \\
\hline Materia verde $\left(\mathrm{kg} / \mathrm{m}^{2)}\right.$ & $3.54^{\mathrm{a}}$ & $2.61^{\mathrm{ba}}$ & $3.07^{\mathrm{ba}}$ & $2.48^{\mathrm{b}}$ \\
Materia seca $\left(\mathrm{kg} / \mathrm{m}^{2)}\right.$ & $0.68^{\mathrm{b}}$ & $0.72^{\mathrm{ba}}$ & $0.98^{\mathrm{a}}$ & $0.93^{\mathrm{ba}}$ \\
$\%$ de materia seca & $19.05^{\mathrm{d}}$ & $27.24^{\mathrm{c}}$ & $32.10^{\mathrm{b}}$ & $37.90^{\mathrm{a}}$ \\
\hline
\end{tabular}

a Tratamiento $1,{ }^{\mathrm{b}}$ Tratamiento $2,{ }^{\mathrm{c}}$ Tratamiento $3,{ }^{\mathrm{d}}$ Tratamiento $4 .{ }^{(\mathrm{a}, \mathrm{b}, \mathrm{c} \text { yd })}$ Letras diferentes en cada columna revelan diferencias entre variables $(\mathrm{p}<0.05)$.

Para la Materia seca mostro que el tratamiento 3 obtuvo el mayor resultado en comparación a los otros tratamientos mostrando una diferencia significativa $(\mathrm{p}<0.05)$, el tratamiento 2 fue igual estadísticamente a los tratamientos 1, 2 y 4 . Flores et al., (2005) encontró resultados para la época de lluvia un rendimiento de pastos asociados de $5,066.0 \mathrm{~kg} / \mathrm{MS} / \mathrm{ha}$, siendo estos resultados menores a los encontrados por el presente estudio en el cual el promedio de producción para el tratamiento 1 fue de 6 , $800.00 \mathrm{~kg} / \mathrm{MS} / \mathrm{ha}$, en el tratamiento 2 de 7 , $200.0 \mathrm{~kg} / \mathrm{MS} / \mathrm{ha}$, tratamiento 3 revelo 
UNIVERSIDAD NACIONAL DE UCAYALI

Revista de Investigación Universitaria

Versión electrónica $2664-8423$

ARTICULO ORIGINAL
Vol. $11 \mathrm{~N}^{\circ} 1$, pp. 475- 482, enero/junio 2021

Recibido 16/01/2021

Aceptado 04/04/2021

Publicado 30/06/2021
9,800.0 $\mathrm{kg} / \mathrm{MS} / \mathrm{ha} . \quad \mathrm{y}$ finalmente el tratamiento 4 obtuvo un rendimiento de 9,300.0 kg/MS/ha; sin embargo estos resultados dependen de varios factores.

Finalmente, en lo que respecta al porcentaje de materia seca el tratamiento 4 mostro el mayor número revelando una diferencia significativa $(\mathrm{p}<0.05)$ entre los tratamientos; estas diferencias significativas se pueden atribuir a las diferentes densidades de siembra, estado fenológico, momento de siembra y cosecha, condiciones atmosféricas y tipo de suelo.

\section{Calidad nutricional}

La tabla 3, contiene la información acerca de los resultados de la composición química de los tratamientos evaluados para la época lluvioso (noviembre a junio) de los tratamientos instalados, donde se revelan que los valores obtenidos para las variables evaluadas que son proteína total (PT), Calcio (Ca), Fósforo (P), fibra detergente neutra (FDN) y fibra detergente acida (FDA).

\section{Proteína total}

El tratamiento 1 tuvo mejores resultados en comparación a los tratamientos 3 y 4 mostrando una diferencia significativa $(\mathrm{p}<0.05)$ para ellos, el tratamiento 2 obtuvo un resultado estadísticamente similar al tratamiento 1. En investigaciones realizados por Flores et al., (2005B) revelo resultados de $19.88 \%$ de proteína total para la época de lluvia y de $10.67 \%$ de PT para la época seca, nuestros resultados se encuentran muy cerca a los mostrados por Flores et al. Estas diferencias de resultados se pueden atribuir a diversos factores como el medio ambiente, calidad de suelo y condiciones atmosféricas.

Tabla 3

Composición nutricional de asociación de pastos permanentes.

\begin{tabular}{lcccc}
\hline \multirow{2}{*}{ Variables } & \multicolumn{4}{c}{$\begin{array}{c}\text { Asociación de leguminosas y } \\
\text { gramíneas }\end{array}$} \\
\cline { 2 - 5 } & $\mathbf{T 1}^{\mathbf{a}}$ & $\mathbf{T 2}^{\mathbf{b}}$ & $\mathbf{T 3}^{\mathbf{c}}$ & $\mathbf{T 4}^{\mathbf{d}}$ \\
\hline Proteína total (N x 6.25) (PT\%) & $15.56^{\mathrm{a}}$ & $15.21^{\mathrm{a}}$ & $6.81^{\mathrm{b}}$ & $5.37^{\mathrm{c}}$ \\
Calcio (Ca\%) & $0.86^{\mathrm{a}}$ & $0.80^{\mathrm{a}}$ & $0.33^{\mathrm{c}}$ & $0.17^{\mathrm{b}}$ \\
Fósforo (P\%) & $0.25^{\mathrm{a}}$ & $0.24^{\mathrm{a}}$ & $0.18^{\mathrm{c}}$ & $0.07^{\mathrm{b}}$ \\
Fibra Detergente Neutra (FDN\%) & $48.85^{\mathrm{a}}$ & $43.82^{\mathrm{b}}$ & $43.34^{\mathrm{b}}$ & $41.89^{\mathrm{c}}$ \\
Fibra detergente acido (FDA\%) & $28.82^{\mathrm{a}}$ & $26.31^{\mathrm{a}}$ & $27.27^{\mathrm{a}}$ & $24.43^{\mathrm{a}}$ \\
\hline
\end{tabular}

a Tratamiento $1,{ }^{\mathrm{b}}$ Tratamiento $2,{ }^{\mathrm{c}}$ Tratamiento $3,{ }^{\mathrm{d}}$ Tratamiento $4 .{ }^{(\mathrm{a}, \mathrm{b}, \mathrm{c} y \mathrm{~d})}$. Letras diferentes en cada columna revelan diferencias entre variables $(\mathrm{p}<0.05)$. Laboratorio de Evaluación Nutricional de Alimentos de la Facultad de Zootecnia de la Universidad Nacional Agraria La Molina.

Minerales

En la composición de calcio y fósforo los tratamientos 1 y 2 mostraron resultados estadísticamente similares y con diferencias significativas $(\mathrm{p}<0.05)$ para los tratamientos 3 y 4 , los resultados obtenidos para el Calcio y fósforo en estudios de Flores et al., 
(2005B) fueron para la época de lluvia de $1.40 \%$ y $0.28 \%$ respectivamente y para la época seca de $0.76 \%$ y $0.19 \%$, mostrando que nuestros resultados se encuentran por debajo por los obtenidos por Flores et al. La composición química del suelo depende mucho para la presencia de los minerales $\mathrm{Ca}$ y $\mathrm{P}$ en la planta.

\section{Fibras}

El contenido de fibras depende mucho de la calidad genética y estado fenológico en el que se encuentre la planta a cosechar siendo así el estado recomendable para cosechar el estado de grano. La fibra detergente neutra (FDN) en el tratamiento 1 mostro un resultado mayor estadísticamente en comparación a los tratamientos 2,3 y 4, los tratamientos 2 y 3 estadísticamente fueron similares. Flores et al., (2005B) revelo resultados de $42.70 \%$ para la época de lluvia y $69.30 \%$ para la época seca; siendo similar a los obtenidos por este estudio. Sin embargo, los resultados obtenidos se encuentran muy elevados para los requerimientos de rumiantes según la NRC (2001), donde menciona que los contenidos adecuados deben estar entre 25 y $35 \%$ de FDN. En la fibra detergente acida (FDA), los resultados obtenidos en el ensayo muestran una diferencia significativa de los tratamientos 1 y 3 en comparación a los tratamientos 2 y 4 siendo estos últimos los de menor contenido, por otro lado, estos contenidos son adecuados para rumiantes ya que la NRC menciona que deben contener de entre 21 a $27 \%$.

\section{Conclusiones}

El tratamiento 1 desarrollo una mayor producción de forraje en materia verde estadísticamente en comparación a los otros tratamientos, con una producción media de $3.54 \mathrm{~kg} / \mathrm{m} 2$, en lo que respecta a la producción de forraje de materia seca los tratamientos 3 y 4 mostraron diferencias estadísticas en comparación a los tratamientos 1 y 2 , con una media de entre 0.98 y $0.93 \mathrm{~kg} / \mathrm{m} 2$ y finalmente, en el porcentaje de materia seca hubo diferencias significativas entre todos los tratamientos.

En la calidad nutricional de las asociaciones el tratamiento 1 y 2 fueron similares en los contenidos de $\mathrm{PT} \%, \mathrm{Ca} \%, \mathrm{P} \%$ y FDA\% y mostrando diferencias estadísticas con los tratamientos 3 y 4 , sin embargo, los contenidos de FDN\% el tratamiento 1 fue estadísticamente diferente a los otros tratamientos.

\section{Agradecimiento}

A la Universidad Nacional Daniel Alcides Carrión, a través del Vicerrectorado de investigación-Instituto Central de Investigación, y los Fondos del Canon y 
regalías mineras. Por el financiamiento del presente estudio.

\section{Referencia bibliográfica}

Aliaga Gutiérrez, Jorge L. 2012. Producción de ovinos. Universidad Nacional Agraria La Molina. Lima, Perú. 299 p.

AOAC. 2005. Official methods of Analysis of AOAC International, 18th edition. AOAC International. Maryland. USA 80:908-912.

Arias Arredondo, Alberto G. 2015. Estudio de la fenología, rendimiento forrajero, y valor nutritivo de dos variedades de avena (Mantaro $15 \mathrm{y}$ Criolla) en los C.E. Casaracra y Alpaicayan - UNDAC, Papana y Huayllay. Tesis de ingeniero Zootecnista. Pasco: Universidad Nacional Daniel Alcides Carrión. Pasco, Perú. 113p.

Calzada, B.J. 1982. Métodos estadísticos para la investigación. Universidad Nacional Agraria La Molina, Lima, Perú. 644p.

Contreras, P.J; De Los Rios B; Montes M y Ramos E.Y. 2013. Consumo y valor nutricional del ensilado de Calamagrostis antoniana Y Avena sativa asociada en diferentes proporciones en alpacas (Vicugna pacos). Revista complutense de ciencias veterinarias 7(1):50-58.

Dulphy, P; Dardillat, M; Jailler, P y Jouany. 1994. Comparison of the Intake and Digestibility of Different Diets in Llamas and Sheep a Preliminary Study. Ann. Zootech. 43: 379-387.

Flores, E.R., J.A Cruz y J. Naupari. 2005A. Utilización de praderas cultivadas en Secano y Praderas Naturales para la Producción Lechera. Boletín Técnico CICCA-FDA-INCAGRO. Lima, Perú.

Flores, E.R; Cruz, J.A y López, M. 2003. Manejo y Uso de los Recursos Genéticos en los Sistemas de Producción Ovina de los Andes Centrales del Perú. Uso y manejo de los recursos genéticos de rumiantes menores en sistemas ganaderos tradicionales en países de Sudamérica. Editado por JP Mueller. FAO-INTA.

Flores, E; Cruz, J y Ñaupari, J. 2005B. Comportamiento Nutricional, Perfil Alimentario y Económico de la Producción Lechera en Praderas Cultivadas en Secano: Caso Pasco. Reporte Científico CICCA-FDAINCAGRO. Lima, Perú. 
INEI, 2012. IV Censo Nacional

Agropecuario. Instituto Nacional de

Estadística e Informática, resultados

generales por departamentos.

Presidencia de la Republica. Lima,

Perú. 62p.

Kuehl, R.O. 2000. Diseño de experimentos.

$2^{\text {da }}$ edición. Thomson Learning,

México D.F. 666 p.

NRC, 2001. Nutrient Requeriments of Dairy

Cattle. 7th ed. National Research

Council. National Academy of

Sciencie. Washington DC, EEUU. $381 \mathrm{p}$.
Rodriguez, M. 2004. Selectividad, consumo y degradabilidad in situ de los pastos naturales de la zona circunlacustre en alpacas. Tesis Médico Veterinario Zootecnista. Puno: Universidad Nacional del Altiplano. Puno, Perú. 41 p.

Van Soest, P.J; Robertson J.B; Lewis B.A. 1991. Métodos de fibra dietetica, detergente neutron, de fibra, y los polisacáridos sin almidón en relación a la alimentación animal. Journal of Dairy Ciencia 74(10): 3583-3597. 